\title{
Thymidine kinase 1 as a tumor biomarker: technical advances offer new potential to an old biomarker
}

\author{
Kiran Kumar Jagarlamudi ${ }^{1,2}$ \& Martin Shaw*,2 \\ ${ }^{1}$ Department of Anatomy, Physiology \& Biochemistry, Swedish University of Agricultural Sciences, VHC, PO Box 7011 , SE 75007 \\ Uppsala, Sweden \\ ${ }^{2}$ AroCell AB, Virdings Allé 32B, SE-754 50 Uppsala, Sweden \\ *Author for correspondence: Tel.: +358 50338 2395; martin.shaw@arocell.com
}

Thymidine kinase 1 (TK1) is a key enzyme in DNA precursor synthesis. It is upregulated during the $S$ phase of the cell cycle and its presence in cells is an indicator of active cell proliferation. In studies since the 1980s, TK1 has been shown as a clinically valuable biomarker for the management of hematological malignancies. However, TK1 activity assays may underestimate serum TK1 in subjects with solid tumors limiting its sensitivity. The development of TK1 immunoassays has made the assay of TK1 more widely available and increased its applicability to solid tumor diseases. This paper will review TK1 as a tumor biomarker with emphasis on recent studies and technologies plus highlight its potential in drug discovery and as a therapeutic target.

First draft submitted: 17 May 2018; Accepted for publication: 2 July 2018; Published online: 2 August 2018

Keywords: anti-TK1 antibodies • biomarker • breast cancer • cell culture • complementary biomarker - Hodgkin • leukemia • lymphoma • proliferation • prostate cancer • solid tumors • TK1 • TK 210 ELISA • translational biomarker

\section{Cell proliferation: what really matters}

Although there are many biomarkers that are related to cell mass and which can be used as an aid to diagnosis, for example PSA in prostate cancer [1] and that can aid in diagnosis and therapy selection, for example BRCA [2], there are few serological biomarkers that indicate the rate of cellular proliferation. Proliferation biomarkers are forward-looking biomarkers and as such could provide especially valuable information for making prognosis and therapy choices. They complement biomarkers of tumor mass as will be described below.

Proliferation biomarkers are proteins that are expressed in proliferating cells and are routinely detected using immunohistological techniques. Such biomarkers include, PCNA, Ki-67 and TK1 [3,4]. These are expressed during the cell cycle in proliferating cells. Ki-67 is the most widely used biomarker of this type and it is expressed during the G1, S, G2 and M phases of cell cycle.

Immunohistological techniques have the drawbacks of being labor intensive, requiring biopsy material and being somewhat subjective. Furthermore, only a small part of the total tumor mass is studied. A serological test that could provide an indication of the total rate of proliferation and disruption would be more convenient and provide unique and valuable information.

TK1 is unique as a proliferation biomarker as it can be determined both immunohistologically in cells and in body fluids using enzyme activity assays and immunoassay.

This article will review recent advances in the application of TK1 as a biomarker and speculate as to how it may be applied in the future development of cancer monitoring and therapies. Emphasis will be laid on the use of immunoassays. Excellent reviews of the earlier literature are available [3,4]. 


\section{Biochemistry \& physical properties of TK1 DNA precursor synthesis}

Thymidine kinases are essential for coupling a phosphate group from ATP to thymidine as a part of the DNA synthesis and repair machinery in all living cells.

There are two major pathways involved in DNA precursor synthesis; the de novo pathway and the salvage pathway. In the de novo pathway, purine and pyrimidine nucleotides are synthesized from low molecular weight precursors, whereas the salvage pathway recycles deoxyribonucleosides (dNs) from degraded DNA. TK1 is a dNs kinase involved in the utilization of thymidine as a part of the salvage pathway [5].

Thymidine kinase activity is present in cells as two isoenzymes: TK1 and TK2.

TK1 converts deoxythymidine (dT) to deoxythymidine monophosphate (dTMP) by transferring a phosphate from ATP. The monophosphate undergoes further phosphorylation to deoxy thymidine triphosphate (dTTP) that is incorporated into DNA. dTTP self-regulates DNA precursor synthesis by negative feedback of TK1 [6].

TK1 activity is cell-cycle regulated and shows a different pattern of activity in normal proliferating cells compared with tumor cells. In normal proliferating cells, TK1 activity reaches peak at late G1 phase/early S phase and is dramatically reduced to undetectable levels by the end of M phase. TK1 activity may be 15 -fold higher in S/G2 compared with G1 [7]. However, TK1 activity may remain elevated in G2 and M phases of cell cycle in malignant cells [8], most likely due to disordered regulation of transcription and degradation. TK2 activity is quite stable without any fluctuations during the cell cycle since it is located in and needed for DNA synthesis in mitochondria [9]. The presence of TK1 in cells is associated with DNA synthesis and cell proliferation, but it is not specific for malignant cells $[3,4]$.

Anticancer therapy, for example, 5-fluorouracil, may suppress the de novo pathway by inhibiting thymidylate synthase providing an advantage for cells more able to use the salvage pathway [10] which may be an explanation for the link between high expression of TK1 and more aggressive malignant disease.

\section{TK1 in DNA repair}

When DNA is damaged by radiation or chemotherapeutic agents, significant increases in TK1 levels are found [5,11]. This may increase the efficiency of the recycling of thymidine released during DNA breakdown. TK1 is involved in DNA repair and depletion of TK1 during DNA recovery results in cell death [5].

\section{Molecular forms of TK1}

TK1 is found in a range of forms depending on the source and purity. The basic intracellular form of TK1 is a homo dimer with a subunit size of $25 \mathrm{kDa}$. The monomer lacks enzyme activity, but in the presence of ATP and/or at high concentrations it polymerizes to dimers and the most active tetramers with a molecular weight of approximately $100 \mathrm{kDa}$ [6]. The intracellular TK1 dimers can be rapidly converted to the active tetramer in the event of DNA damage, in advance of new synthesis [12].

TK1 is released from cells that die during proliferation; the concentration of TK1 in extracellular fluid is a measure of disruption of dividing cells. Normal cells seldom disintegrate during proliferation and it usually only occurs during accelerated or unregulated proliferation, as in malignancies. Following its release, TK1 forms complexes of differing molecular weights and enzyme activities, a factor which is of importance regarding the development of assays.

Cellular TK1 from human leukemia cells eluted mainly as tetramers $(100 \mathrm{kDa})$ with a minor peak around 700 $\mathrm{kDa}[13,14]$. However, serum TK1 from a leukemia patient eluted as a high MW complex around $700 \mathrm{kDa}$ plus a minor peak of low molecular weight [14,15]. Analysis of molecular forms of serum TK1 from breast cancer and prostate cancer patients has demonstrated the existence of active TK1 as complexes ranging in size from 720 to $200 \mathrm{kDa}$. Furthermore, immunoblot analysis of serum TK1 showed that it also included low molecular weight complexes that lacked enzyme activity [15].

\section{TK1 assays}

Because of its close association with DNA synthesis and cell proliferation, TK1 is a valuable biomarker for studying malignancies. TK1 has been quantified by two different technologies: by measuring its enzyme activity or its mass, via immunoassay. A brief description of the technologies is given below and summarized in Table 1.

Intracellular active TK1 is a tetramer but upon release it polymerizes producing high molecular weight complexes with a range of molecular weights and enzyme activities (see Figures $1 \& 2$ ) [15]. Furthermore, serum may contain 
enzyme inhibitors [16]. These factors provide challenges to the assay of TK1, particularly for the forms associated with solid tumors [15].

\section{Enzyme activity-based assays}

TK1 activity assays are based on the transfer of phosphate groups from ATP to labeled TK1 substrates resulting in labeled monophosphate derivatives (dTMP) which are detected by a variety of methods.

\section{$T K$-radio enzymatic assay (TK-REA)}

TK-REA uses ${ }^{125}$ I-labeled deoxyuridine as a substrate. In brief, radioactive substrate is added to the sample and incubated at $37^{\circ} \mathrm{C}$. TK1 in the sample converts the labeled substrate to ${ }^{125} \mathrm{I}$-deoxyuridine monophosphate (dUMP). Then the reaction product (dUMP), but not the unconverted substrate, is absorbed by a TK-REA separator tablet. After washing, the radioactivity in the sedimented particles is measured and TK1 levels expressed as U/1 [17]. The higher the radioactivity the higher the TK1 activity.

\section{TK-Liaison assay}

This assay utilizes a two-step procedure where in the first step, TK1 in the sample phosphorylates 3-azido-3deoxythymidine (AZT) to the corresponding 5-monophosphate (AZTMP).

In the second step, the generated AZTMP is detected utilizing a competitive ELISA technique. In brief, the sample is incubated with goat anti-AZTMP antibodies and HRP-labeled AZTMP in a microtiter plate coated with an excess of rabbit antigoat antibodies. Competition between the generated AZTMP and the HRP-labeled AZTMP occurs for the primary goat antibody. The goat antibodies, with bound AZTMP or HRP-labeled AZTMP, are captured by the antigoat antibodies on the microtiter plate. Unbound HRP-labeled AZTMP is washed away, and the bound HRP-labeled AZTMP is quantified using TMB as a substrate. The color developed is inversely proportional to the initial TK1 activity and is expressed in $\mathrm{U} / 1$ [18].

\section{$\left.{ }^{3} \mathrm{H}\right]$-deoxythymidine phosphorylation assay}

The assay is based on the use of tritiated deoxy-thymidine, a natural substrate for TK1 instead of substrate analogs [19]. It may be considered the 'gold standard' for determining TK1 activity. In brief, samples are incubated with a reaction mixture containing ATP, DTT, $\mathrm{MgCl}_{2}$ and ${ }^{3} \mathrm{H}$-labeled deoxy-thymidine. TK1 in the sample converts the labeled substrate into tritiated deoxy thymidine monophosphate $\left({ }^{3} \mathrm{H}\right.$-dTMP). The product of the reaction is spotted onto DE-81 filter papers and the filter papers allowed to dry. The filter paper is then washed with ammonium formate to remove the unconverted substrate. The bound ${ }^{3} \mathrm{H}$-dTMP is eluted from the filter papers using $\mathrm{HCl}$ and $\mathrm{KCl}$ buffer and its radioactivity measured using $\beta$-scintillation counter. The activity is expressed as $\mathrm{pmol} / \mathrm{min} / \mathrm{ml}$. The higher the radioactivity, the higher the TK1 activity in the sample.

\section{Divitum assay}

The Divitum TK1 enzyme activity assay utilizes bromodeoxyuridine (BrdU) as a substrate. In this assay, TK1 in the sample phosphorylates BrdU to bromodeoxyuridine monophosphate (BrdUMP). This BrdUMP undergoes further phosphorylation by added yeast enzymes and the formed bromodeoxyuridine triphosphate (BrdUTP) is incorporated, via a DNA polymerase, into DNA strands that are bound to a microtiter plate. The BrdUTP bound to the plate is then quantified using a bromodexoyuridune antibody conjugated with alkaline phosphatase. The color developed is proportional to the amount of bromodeoxyuridine monophosphate formed by TK1 and is, therefore, proportional to the initial TK1 activity. TK1 activity is expressed in Divitum units/l (Du/l) [20]. The Divitum assay is optimized for measuring TK1 activity in high molecular weight complexes and may be less sensitive than the Liaison method for measuring low MW TK1 complexes [21], those that also predominate in serum from subjects with solid tumors.

\section{Immunoassays}

The development of antibodies against different exposed regions of TK1 demonstrated the potential for the development of highly effective TK1 immunoassays. Moreover, immunoassays widen the clinical applications of TK1 for diagnosis and treatment monitoring of different solid tumors by enabling determinations of TK1 forms that lack enzyme activity. 


\section{TK1 immunoassays}

Several attempts have been made to develop TK1 ELISAs suitable for determining TK1 concentrations in serum samples [22], but none of these are widely available.

One reason is the need for antibodies of appropriate specificities, in particular the development of antibodies against the exposed '210' epitope covering the C-terminal amino acid sequence 194-225 [23]. Two assays have been developed based on antibodies against this epitope; a direct dot-blot assay with chemiluminescence end point [24] and a microtiter sandwich ELISA [25].

\section{Dot-blot chemiluminescence assay}

This dot-blot assay is a nitrocellulose membrane based assay with a chemiluminsecent substrate utilising a primary chicken IgY antibody directed against a C-terminal TK1 epitope and a secondary labeled anti-IgY antibody. In brief, the sample is spotted onto a nitrocellulose membrane where the proteins in the sample bind. After blocking, the membrane is incubated with a primary anti-TK1 antibody which binds to the TK1 on the membrane. After washing, a biotinylated second antibody directed against IgY antibodies is added followed by streptavidin labeled HRP and a chemiluminescent substrate. The light intensity of the dots is measured by a CIS-II imaging system. The greater the emission of light, the higher the TK1 concentration in the sample. TK1 levels are expressed as $\mathrm{pM}$ [24].

\section{TK 210 ELISA}

A problem in the development of TK1 assays has been that TK1 circulates in serum in complexes of differing molecular weights and enzyme activities. Furthermore, the molecular weight distributions of these complexes varies between different types of malignancies [15].

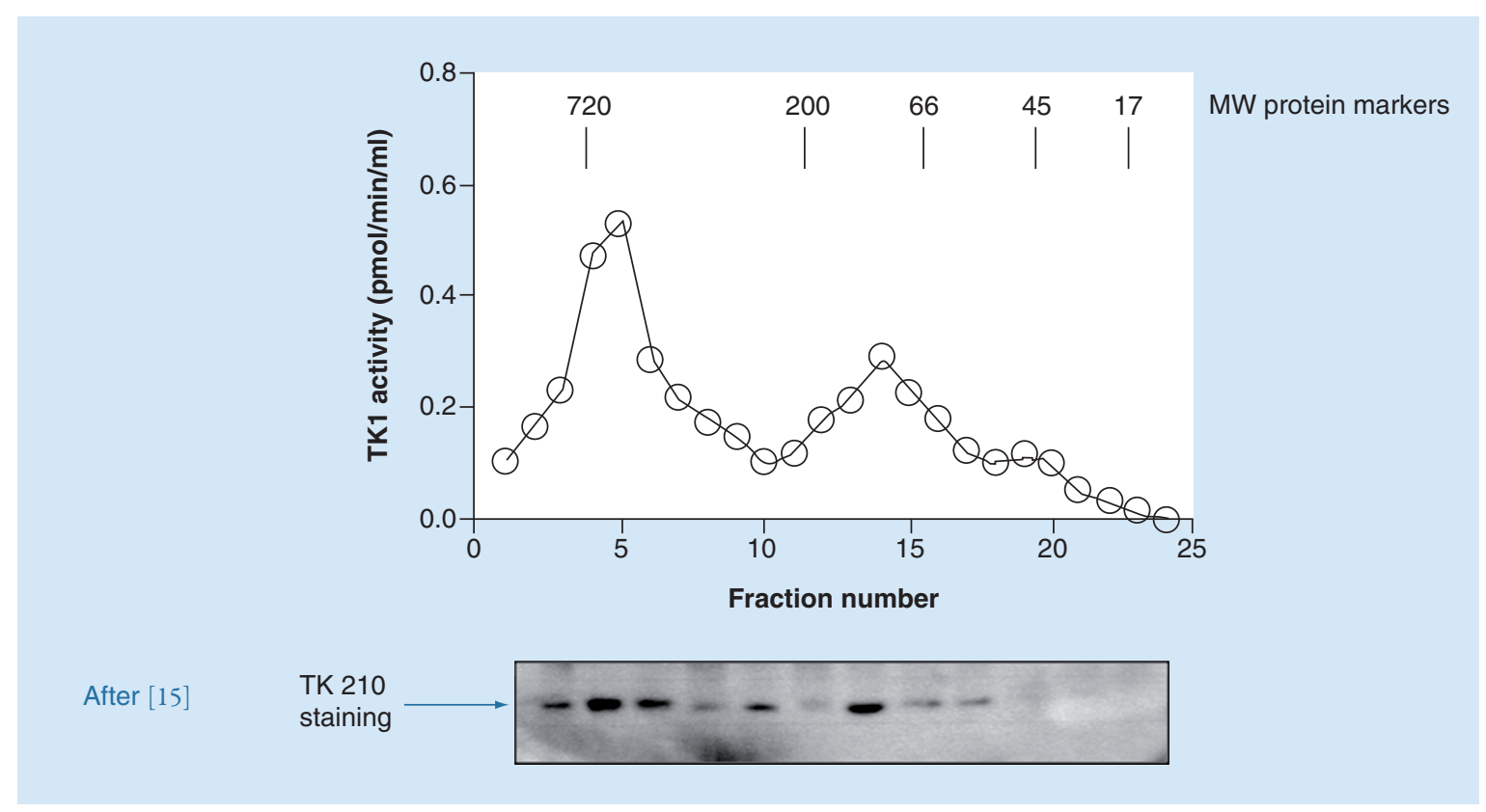

Figure 1. TK1 complexes, immunoreactivity and enzyme activities in serum from a subject with myelodysplasia.

The AroCell TK 210 ELISA system has reduced this problem by pre-incubating the sample with a buffer containing agents that breakup the high molecular weight complexes. Following this pretreatment of the sample, the TK1 is assayed using a standard microtiter ELISA technique. In brief, the treated samples are added to a microtiter plate coated with anti TK 210 monoclonal antibodies. After incubating and washing, a second anti-TK 210 antibody labeled with biotin is added. After further washing, the color is developed with streptavidin labeled horse radish peroxidase with TMP as substrate. The stronger the color generated, the higher the TK1 concentration in the sample. The values are expressed as $\mu \mathrm{g} / \mathrm{l}[25]$. 


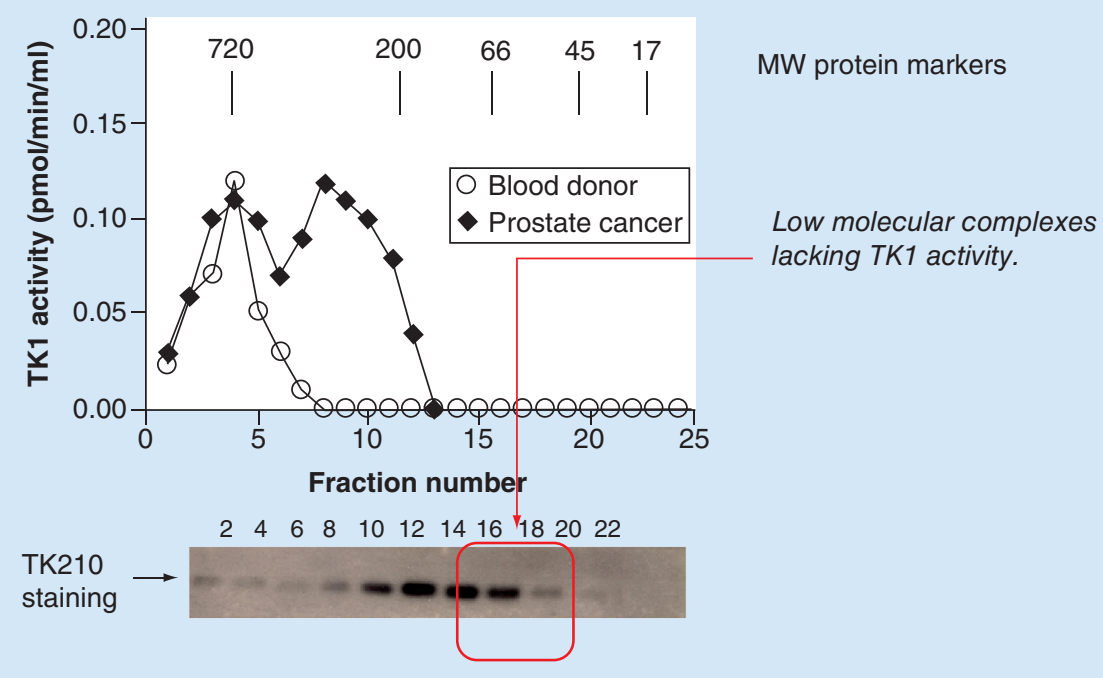

Figure 2. TK1 complexes, immunoreactivity and enzyme activities in serum from a subject with prostate cancer.

\begin{tabular}{|c|c|c|}
\hline Assay variable & TK1/TK 210 immunoassays & TK1 enzyme activity assays \\
\hline \multirow[t]{7}{*}{ Applicability } & + Greater sensitivity in subjects with solid tumors [16]. & $\begin{array}{l}\text { - Not all TK1 is enzymatically active in sera from subjects with solid } \\
\text { tumors [15]. }\end{array}$ \\
\hline & + More consistent TK1 values in different disease types. & - Specific activity of serum TK1 may differ between disease types [15]. \\
\hline & - Potentially, a different antibody for each species. & + Potentially applicable to many animal species and cell lines. \\
\hline & $\begin{array}{l}\text { + Species specific differences in TK } 210 \text { antigen may make it } \\
\text { possible to distinguish TK1 from host and implant in xenograft } \\
\text { studies. }\end{array}$ & - Source of TK1 activity may be unclear in xenograft studies. \\
\hline & $\begin{array}{l}\text { + Probable good correlation with activity assays in sera from } \\
\text { hematological malignancies. }\end{array}$ & $\begin{array}{l}\text { + Probable good correlation with immunoassays in sera from } \\
\text { hematological malignancies. }\end{array}$ \\
\hline & + Potential to use the same antibody for immunohistology. & - \\
\hline & + Probable good correlation with activity assays in cell extracts. & + Probable good correlation with immunoassays in cell extracts. \\
\hline \multirow[t]{3}{*}{ Confounding factors } & + Defined reaction; less assay interference. & - Serum may contain enzyme inhibitors [16]. \\
\hline & - Antibody dependent. & + Not dependant on a specific antibody. \\
\hline & - Differences in unitage between antisera. & - Differences in unitage between substrates. \\
\hline
\end{tabular}

Figures $1 \& 2$ show examples of the relationship between the presence of the TK 210 epitope and enzyme activity in different molecular weight fractions in serum from subjects with different malignancies. Note that in a sample from a subject with a hematological malignancy there is a good agreement between the presence of the TK 210 epitope, as shown by Western blotting, and enzyme activity whereas much of the TK 210 in a subject with a solid tumor was associated with low molecular weight fractions with low or no activity [15]. This finding helps to explain the predominance of studies on the application of TK1 activity assays being in the field of hematological malignancies. The pretreatment of the samples may make comparisons between subjects and types of malignancies more reproducible. The development of immunoassays based on the TK 210 antigen opens new possibilities for the study of TK1 in solid tumors and, most likely, also in different forms of hematologic malignancies.

\section{TK1 in biomarker studies}

The rate of cellular proliferation and disruption is an important parameter usually monitored by immunohistological studies on biopsies. The 'gold standard' is the proliferation index which is the \% of cells staining for Ki-67. Tissues that have a high $\mathrm{Ki}-67$ proliferation index tend to also have a high proliferation index based on TK1 staining, although the same cells do not necessarily stain for both biomarkers [26,27]. TK1 staining may be better than Ki-67 at discriminating between breast cancer stages [28]. 
However, the main advantage of TK1 as a proliferation biomarker is being accessible in serum and, therefore, providing an indication of the total disease activity in the subject. In this, it may be unique as a serological biomarker for cell proliferation and disruption.

\section{TK1 in population screening}

Serum TK1 may be elevated in many situations where increased cell proliferation may be occurring. This includes a range of conditions including infections, inflammatory conditions and (pre) malignancies [3,4,17]. A large-scale population screen utilizing a dot-blot chemiluminescent assay found that $83 \%$ of subjects with an elevated serum TK1 ( $>2$ pM, highest 1.1 percentile) had an increased risk of having a pathological condition, $22 \%$ with viral infections or inflammation and 61\% malignancies [29]. An elevated serum TK1 may be a signal for further clinical investigation, without indicating the cause.

\section{TK1 \& hematological malignancies}

Probably because most of the serum TK1 in hematological malignancies is enzymatically active (see Figure 1), serum TK1 has been found to be a valuable biomarker in the management of hematological malignancies. Furthermore, lymphocytes from subjects with lymphomas probably express a higher level of TK1 than those from normal subjects [30].

Studies have shown that in hematological malignancies, serum TK1 activity levels:

- are associated with clinical stage;

Subjects with apparently early clinical stage disease but with a relatively elevated serum TK1 activity had a tendency for disease progression [31];

- are associated with tumor burden [30];

- correlate with expression of the proliferation biomarker Ki-67 [31,32];

- may correlate with the total mass of proliferating cells (Ki-67 index $\times$ tumor mass) [30];

- may add value to other tumor-related biomarkers [33];

- can aid in making prognoses [33-35];

- may be a biomarker of disease progression [36].

There have been, as of yet, fewer studies on hematological malignancies where serum TK1 has been determined using immunoassays. However, TK1 measurements using a dot-blot chemiluminescence assay prior to and after therapy were shown to correlate with clinical response and therapy in non-Hodgkin's lymphoma [37] and aid in making prognoses in subjects with chronic lymphocytic leukemia [38].

Furthermore, serum TK1 levels measured using a TK 210 immunoassay correlated closely with TK1 activity levels measured using the tritiated thymidine assay and they gave a similar receiver operating characteristic (ROC) curve ( 0.84 for TK 210 ELISA, 0.79 for ${ }^{3} \mathrm{H}$-deoxythymidine phosphorylation assay) for distinguishing between subjects with and without hematological malignancies [39]. Preliminary studies indicate that immunoassay of TK1 using the TK 210 antibody can distinguish between healthy subjects and those with hematological malignancies and possibly aid in disease monitoring [40,41].

The close agreement between results with activity and immunological assays is valuable in that it enables results with more modern assays to be compared with the historical data. For further references on this topic, the reader is referred to references [3,4].

\section{TK1 in the study of solid tumors}

There have been fewer studies on the use of serum TK1 activity to study solid tumors although there are many studies showing increased expression of TK1 in solid malignancies [3,4] and even in pre-malignant conditions [42]. This may be due to the greater difficulty of measuring TK1 activity in serum from subjects with solid tumors as mentioned previously. This review will mainly concentrate on results obtained using immunoassay with reference, where appropriate, to studies performed with other methods.

Elevated serum TK1 activities or immunological concentrations have been found in many solid tumors including gastric [43], ovary [44], cervical [45], esophageal [46], lung [22,47], prostate [15,48] and breast [15,42]. Elevated serum TK1 levels post surgery have been associated with poorer outcome [49]. 

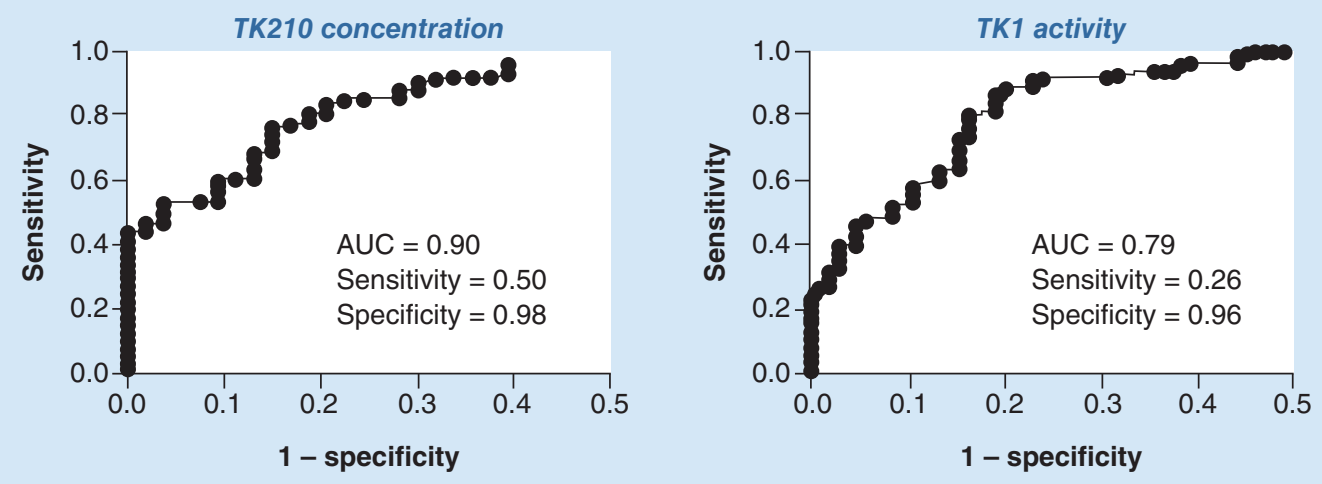

After [25]

Figure 3. Receiver operating characteristic analyses of serum TK 210 ELISA and TK1 activity assays for distinguishing breast cancer patients from healthy subjects.

AUC: Area under the curve.

Figure 4. Receiver operating characteristic analyses of

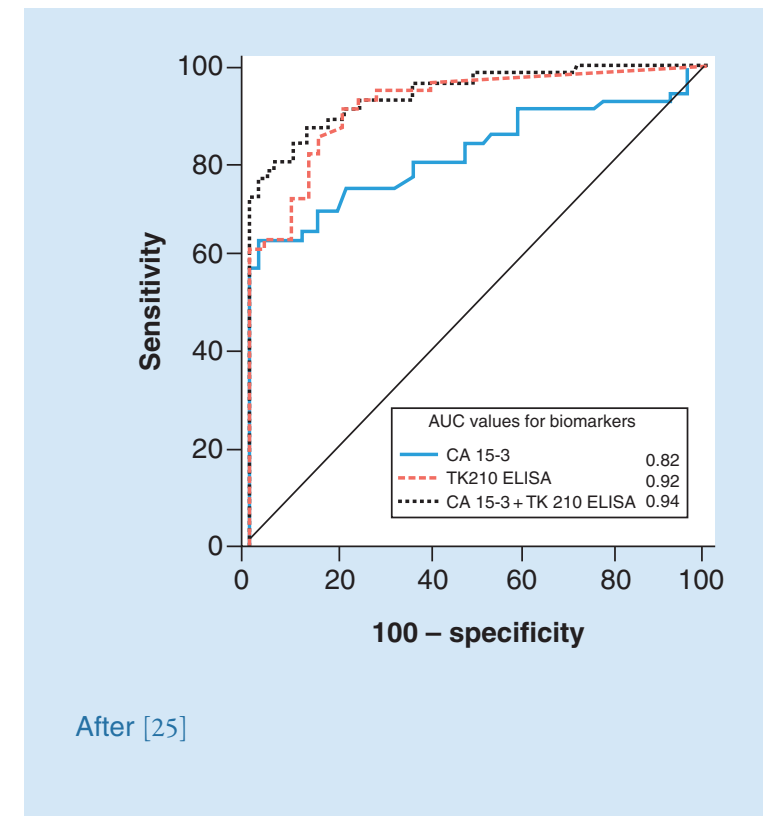
serum TK 210 ELISA and CA15-3 for distinguishing breast cancer (T2) patients from healthy subjects.

Studies have shown that in solid malignancies, serum TK1 immunoassay levels:

- are associated with clinical stage [25,49];

Subjects with apparently early clinical stage disease but with a relatively elevated serum TK1 activity had a tendency for disease progression [49];

- may add value to other tumor-related biomarkers [25,48,50];

- can aid is in making prognoses;

Elevated serum TK1 levels post surgery and chemotherapy have been associated with poorer outcome [49];

- may be a biomarker of disease progression [49].

The greater sensitivity for immunoassay in studying solid tumors may be explained by the occurrence of enzymatically inactive TK1 forms in the serum of subjects with solid tumors [15]. Figure 3 compares the diagnostic accuracy (ROC analyses) of TK1 an enzyme activity assay and TK 210 ELISA for the diagnosis of stage II breast 
cancer. The TK 210 ELISA was nearly twice as sensitive ( 0.50 vs 0.26$)$ with a similar specificity and a higher AUC (0.90 vs 0.79) [25]. Other studies have shown TK1 immunoassay to be more sensitive than enzyme activity assays in studying solid tumors $[16,47]$.

TK1 immunoassay can be valuably combined with other biomarkers. Jiang et al. found that adding TK1 immunoassay to a panel of biomarkers increased the diagnostic accuracy for lung cancer [50]. Similarly, the addition of TK 210 ELISA to CA15-3 increased the ROC value for diagnosing breast cancer (Figure 4) [25] and its addition to pro-PSA and the prostate health index increased the ROC value for the diagnosis of prostate cancer [48].

\section{TK1 as a therapeutic target}

TK1 is not essential for normal cell growth but it is important in the repair of DNA damage such as may be caused by chemotherapy [6]. Furthermore, upregulation of TK1 can reduce the efficacy of chemotherapy, for example by 5-fluoro-2'-deoxyuridine (5FdUrd) [10].

Anticancer drugs (e.g., FdUrd) may inhibit the de novo pyridine pathway which leads to toxicity for normal tissue while, possibly, selecting for malignant tissues with a higher TK1 expression.

Drugs that require TK1 for activation have, therefore, the potential to be less toxic to normal cells while targeting malignant tissues with higher TK1 concentrations.

This has been shown using a radioiodine labeled thymidine derivative, $\left[{ }^{123 / 125} \mathrm{I}\right]^{-} 5$-iodo- $4^{\prime}$ thio-2'-deoxyuridine, which is incorporated into DNA resulting in cell death. To increase the incorporation of this radioactive thymidine derivative into DNA, de novo synthesis of thymidine was blocked via the application of the thymidine synthetase inhibitor FdUrd [51].

Boron $\left({ }^{10} \mathrm{~B}\right)$ has the property of emitting very short-range radiation when bombarded with neutrons killing the cells in which it is found but sparing surrounding tissue. Several boron labeled thymidine derivatives have been developed and shown to be valuable in the radio-therapy of tumors, especially gliomas. The higher TK1 content of proliferating tumors may make them particularly sensitive to ${ }^{10} \mathrm{~B}$ assisted radiotherapy. For example, in a study comparing TK1 knock-out and wild-type tumors implanted into rats, the wild-type $(\mathrm{TK} 1+)$ tumors took up about 2.5-times more boron than the TK1- knockout tumors. Animals bearing TK1+ tumors had an average 15-fold reduction in mean tumor volume on day 30 after implantation compared with TK1- tumors [52].

TK1 has been reported to be expressed on the surface of Burkitt's lymphoma and acute lymphoblastic leukemia cells, but not on healthy cells, making it a potential target for immunotherapy [53].

Assays determining TK1 concentrations in tumor tissues and, maybe, in serum could help to select those subjects most likely to benefit from these novel types of therapies.

\section{TK1 in positron imaging}

Positron $\left[{ }^{18} \mathrm{~F}\right]$ labeled thymidine derivatives (e.g., $3^{\prime}$-deoxy- $3^{\prime}\left[{ }^{18} \mathrm{~F}\right]$-fluorothymidine $\left[\left[{ }^{18} \mathrm{~F}\right] \mathrm{FLT}\right]$ ) offer a means of imaging tumor mass and tumor proliferation. These compounds are phosphorylated by TK1 and are then trapped in the cells [54]. That malignant cells tend to have higher concentrations of TK1 makes these derivatives particularly attractive.

In general, the uptake of $\left[{ }^{18} \mathrm{~F}\right]$ thymidine derivatives correlate better with the proliferation index in cell lines with greater dependence on the salvage pathway [55].

Therapy with agents that suppress the de novo pathway (e.g., hydroxyurea) or cause DNA damage may cause upregulation of TK1 which leads to an initial increase in $\left[{ }^{18} \mathrm{~F}\right]$ FLT uptake (flare) $[12,56]$ followed by a decline as cell death occurs. Uptake may also differ according to the dependence of the tumor on the de novo or salvage thymidine pathways [57]. Thus, positron imaging with $\left[{ }^{18} \mathrm{~F}\right]$ thymidine derivatives could potentially provide information as to the mechanism and time course of therapeutic effects, but it could also mean that the optimal time to image a tumor may vary according to the mechanism and pharmacokinetics of the agent $[56] .\left[{ }^{18} \mathrm{~F}\right]$ thymidine derivatives uptake can be used to study cell proliferation in both in vitro culture [12,58] and xenografts [59] making it a translatable biomarker.

Thymidine kinase in drug discovery and development In vitro studies

A crucial stage in drug discovery is in vitro validation of potential new compounds. 
TK1, being involved in DNA synthesis and repair [5], provides an in vitro means of measuring the effects of drugs that target these pathways, TK1 immunohistology could aid in the selection of cell lines with different expression of TK1 which may respond differently to anti-metabolites [60].

TK1 immunoassay in cell culture can be used to follow dose-effect curves of drug-cell interactions. For example, in a leukemic cell line treated with doxorubicin, low doses of doxorubicin induced intracellular TK1 concentrations but at higher drug levels it decreased and, simultaneously, TK1 appeared in the culture medium as cell death occurred [61]. These patterns match what is known about doxorubicin, in that it damages DNA and that TK1 is involved in DNA repair. Furthermore, this pattern is similar to that seen also in positron imaging where an increased uptake of positron labeled $\left[{ }^{18} \mathrm{~F}\right]$ thymidine analogs occurs in the early stages of therapy (flare) then declining as treatment progresses [57]. This may be particularly observed with inhibitors of the de novo pathway [12].

Since TK1 is involved in DNA repair and resistance to chemotherapy, increases in TK1 may not be seen if compounds which suppress cell proliferation by other means - for example, anti-hormonal therapy or CDK4/6 inhibition, are used. This has been shown for the CDK 4/6 inhibitor Palbociclib [62] and anti-estrogen therapy [63] using the Divitum enzyme activity assay.

Very few cells are needed for a study and together with the ability to automate ELISA procedures, new compounds can be rapidly and economically tested. Furthermore, TK1 is an early indicator of cellular proliferation and may be upregulated earlier than Ki-67 and before BdrU incorporation into DNA occurs [64].

\section{Xenograft studies}

Potential therapeutic compounds are often studied using tumor xenografts in immune-deficient mice. TK1 activity assays will measure TK1 derived from both the human graft and the host. However, the human TK 210 antigen differs from that in mice, potentially, enabling better discrimination of the effects of the test substance on the tumor graft than using TK1 activity assays.

\section{Clinical studies}

In human clinical trials, serum and tissue TK1 could provide a means to stratify patients/subjects according to the clinical stage of their disease and the rate of cell proliferation and disintegration [25,46]. This could enable clearer discrimination between study groups and also clearer definition of the treatment effects.

Elevated tissue TK1 values are associated with a poorer therapeutic prognosis [65] and may provide a guide to therapeutic sensitivities, for example, subjects with a high tumor TK1 responded less well to tamoxifen therapy [65].

During therapy, serum TK1 levels may fluctuate, due to destruction of tumor cells but also, possibly, due to the proliferation and destruction of normal cells as a part of an inflammatory response or general cytotoxic effect [66]. This is supported by findings of elevated serum TK1 levels following surgical removal of tumors [67]. In general, therefore, a therapeutic response is associated with an increase in serum TK1 concentration during and immediately after therapy. However, approximately 3-5 weeks after completed successful therapy TK1 concentrations should be reduced to near normal concentrations [37,68]. A much faster apparent decline may be seen if TK1 enzyme activity is measured [69]. This decline could possibly be due to the presence of TK1 inhibitors in serum that inhibit its activity but leave it open for immunoassay [16].

If serum TK1 protein is high and/or increasing it is often associated with relapse or therapeutic escape [36].

Compounds that are not directly cytotoxic but reduce cell proliferation may reduce TK1 levels. Check point inhibitors that prevent cells entering the $S$ phase of cell proliferation may inhibit TK1 production, therefore, a positive response could be linked with a fall in TK1. This was shown for the CDK4/6 inhibitor Palbociclib using the Divitum TK1 activity assay [62]. Similarly, successful anti-hormonal therapy of breast cancer may be associated with a fall in serum TK1 activity, with decreased TK1 activity levels after a month being associated with better outcome [63].

\section{Translational biomarker}

A critical parameter in studying the effects of anticancer therapy is the proliferation rate of the malignancy. Assays of tissue and serum TK1 can be used to study the effects of compounds in cell-culture, potentially in xenografts and in human clinical trials making it a translational biomarker. 


\section{Future perspective}

The development of better immunoassay techniques for TK1 based on the 210 epitope opens new possibilities for the application of this biomarker.

The development of anticancer therapies and tumor biomarkers are being rapidly advanced due to greater understanding of the genetic and biological factors that stimulate and inhibit tumor growth. The rapid progress in cancer immunotherapy is offering new hope and possibilities in treatment of previously incurable malignancies, but the very high cost of novel therapies, often in the range of hundreds of thousands of dollars for a single patient, plus the risk of side effects, are necessitating improved patient selection. Thus, biomarkers will have an increasingly important role in future personalized medicine.

However, there is a problem in that there is a lack of serological biomarkers that can provide forward-looking information regarding the effects of therapy (prognostic biomarkers). For example, the American Society of Oncologists wrote that "No biomarkers for monitoring advanced breast cancer are available" [70] and in prostate cancer, the European Association of Urology have stated "PSA is a less reliable biomarker in monitoring advanced metastatic disease" [71].

TK1 has great potential here since it is a biomarker for the turnover of proliferating cells and may aid selection of the patients with aggressive disease and most in need of therapy. Thus, a TK1 protein assay can be said to 'measure what really matters'. It could save considerable resources and avoid unnecessary side-effects in those less likely to benefit from a particular therapy.

Since most malignant cells tend to express TK1 at higher and unregulated concentrations than normal cells, TK1 has the potential to monitor therapies across a wide range of cancer types and therapies.

TK 210 immunoassays increase the range of malignancies that can be studied with this biomarker, plus immunoassays are more compatible with standard laboratory assay platforms. This simplifies the combining of TK1 assays with other biomarkers leading to the development of new and better algorithms for patient diagnosis and monitoring.

The relative overexpression of TK1 in proliferating cells makes TK1 an interesting target for therapeutic intervention and imaging probes. The role of TK1 in DNA damage repair suggests that its assay could add particular value to the study of anti-metabolic agents and indicates its potential as a complementary diagnostic.

Cell proliferation and disruption are key parameters in oncology, TK1 immunoassays offer new, improved means of monitoring these essential processes, in model systems pharmaceutical research and clinical practice.

\section{Executive summary}

TK1 is a well-validated biomarker for studying malignancies. However, it is not widely applied due to complicated and closed assay systems and lack of studies utilizing recent analytical methods. However:

- the immunoassay of TK1 increases its value as a biomarker.

- the immunoassay of TK1 makes it a more sensitive and novel biomarker for studying solid malignancies.

- TK1 levels in malignancies are associated with clinical stage offering potential for patient and subject stratification.

- elevated and/or increasing serum TK1 levels after treatment indicate therapy escape.

- TK1 may be a particularly valuable biomarker for monitoring therapy with cytotoxic agents.

- TK1 can be used as a translational biomarker in drug discovery.

- changes in cell culture seem to be similar to those seen in vivo and may offer a new biomarker for studying anti-proliferative agents.

- TK1 has great potential as a complementary biomarker.

- changes with disease status and response are associated with changes in TK1 levels.

- TK1 may have potential as a target for novel therapeutics and imaging agents.

- there is need for better biomarkers to follow therapy response.

- TK1 levels are associated with changes in the disruption of proliferating cells.

- TK1 may be profitably combined with other biomarkers to improve diagnostic accuracy. 
Financial \& competing interests disclosure

KK Jagarlamudi is employed by AroCell AB. M Shaw is an independent consultant contracted to AroCell AB. The authors have no other relevant affiliations or financial involvement with any organization or entity with a financial interest in or financial conflict with the subject matter or materials discussed in the manuscript apart from those disclosed.

No writing assistance was utilized in the production of this manuscript.

\section{Acknowledgements}

The authors acknowledge with thanks Staffan Eriksson for his valuable contributions to this manuscript, and Kris Overby, CMPP for editorial support.

\section{Open Access}

This work is licensed under the Attribution-NonCommercial-NoDerivatives 4.0 Unported License. To view a copy of this license, visit http://creativecommons.org/licenses/by-nc-nd/4.0/

\section{References}

Papers of special note have been highlighted as: • of interest; $\bullet \bullet$ of considerable interest

1. Tsodikov A, Gulati R, Heijnsdijk EAM et al. Reconciling the effects of screening on prostate cancer mortality in the ERSPC and PLCO trials. Ann Intern Med.167(7), 449-455. (2017).

2. Tung NM, Garber JE. Review article. BRCA1/2 testing: therapeutic implications for breast cancer management. British J. Cancer 119(2), 141-152 (2018).

3. O’Neill KL, Buckwalter M, Murray BK. Thymidine kinase: diagnostic and prognostic potential. Expert. Rev. Mol. Diagn. 1(4), 428-433 (2001).

-. An excellent review of the early literature on the basic biochemistry and application of TK1 in studying tumor biology.

4. Topolcan O, Holubec Jr L. The role of thymidine kinase in cancer diseases. Expert Opin. Med. Diagn. 2(2), 129-141 (2008).

-• An excellent review of the early literature on the basic biochemistry and application of TK1 in studying tumor biology.

5. Chen Y-L, Eriksson S, Chang Z-F. Regulation and functional contribution of thymidine kinase 1 in repair of DNA damage. The J. Biol. Chem. 285(35), 27327-27335, (2010).

-. Basic information on the role of TK1 in DNA damage and repair.

6. Munch-Petersen B. Enzymatic regulation of cytosolic thymidine kinase 1 and mitochondrial thymidine kinase 2: a mini review. Nucleosides Nucleotides Nucleic Acids 29(4-6), 363-369. (2010).

7. Sherley JL, Kelly TJ. Regulation of human thymidine kinase during the cell cycle. J. Biol. Chem. 263(17), 8350-8358 (1988).

- Information on changes in TK1 concentration and activity during the cell cycle.

8. Chang ZF, Huang DY. The regulation of thymidine kinase in HL-60 human promyeloleukemia cells. J. Biol. Chem. 268(2), 1266-1271 (1993).

9. Berk AJ, Clayton DA. A genetically distinct thymidine kinase in mammalian mitochondria. Exclusive labeling of mitochondrial deoxyribonucleic acid. J. Biol. Chem. 248(8), 2722-2729 (1973).

10. Fanciullino R, Evrard A, Cuq P et al. Genetic and biochemical modulation of 5-fluorouracil through the overexpression of thymidine kinase: an in-vitro study. Anticancer Drugs17(4), 463-470. (2006).

11. Haveman J, Sigmond J, van Bree C, Franken NA, Koedooder C, Peters GJ. Time course of enhanced activity of deoxycytidine kinase and thymidine kinase 1 and 2 in cultured human squamous lung carcinoma cells, SW-1573, induced by gamma-irradiation. Oncol. Rep. 16(4), 901-905 (2006).

12. Chen X, Yang Y, Berger I et al. Early detection of pemetrexed-induced inhibition of thymidylate synthase in non-small-cell lung cancer with FLT-PET imaging. Oncotarget 8(15), 24213-24223 (2017).

13. Karlström AR, Neumüller M, Gronowitz JS, Källander CF. Molecular forms in human serum of enzymes synthesizing DNA precursors and DNA. Mol. Cell Biochem. 92(1), 23-35. (1990)

14. Hanan S, Jagarlamudi KK, Liya W, Ellen H, Eriksson S. Quaternary structures of recombinant, cellular and serum forms of thymidine kinase 1 from dogs and humans. BMC Biochem. 13(12), 6-8 (2012).

-. Basic data on the relationship between TK1 complexes of different MWs and their enzyme activity.

15. Jagarlamudi KK, Hansson LO, Eriksson S. Breast and prostate cancer patients differ significantly in their serum thymidine kinase 1 (TK1) specific activities compared with those hematological malignancies and blood donors: implications of using serum TK1 as a biomarker. BMC Cancer 15(66), 7-9 (2015).

-• Basic data comparing the MW and specific activities of serum TK1 complexes in serum from subjects with hematological malignancies and solid tumors. 
16. He Q, Zhang P, Zou L et al. Concentration of thymidine kinase 1 in serum (S-TK1) is a more sensitive proliferation marker in human solid tumors than its activity. Oncol. Rep. 14(4), 1013-1019 (2005).

-• Indicates that serum may contain inhibitors of TK1 and immunoassay may be more sensitive than enzyme activity assays for measuring TK1 in serum from subjects with solid tumors.

17. Gronowitz JS, Källander FR, Diderholm H, Hagberg H, Pettersson U. Application of an in vitro assay for serum thymidine kinase: results on viral disease and malignancies in humans. Int. J. Cancer 33(1), 5-12. (1984).

18. Öhrvik A, Lindh M, Einarsson R, Grassi Eriksson S. Sensitive nonradiometric method for determining thymidine kinase 1 activity. Clin. Chem. 50(9), 1597-1606 (2004).

19. Munch-Petersen B. Differences in the kinetic properties of thymidine kinase isoenzymes in unstimulated and phytohemagglutinin-stimulated human lymphocytes. Mol. Cell. Biochem. 64, 173-185 (1984).

20. Gronowitz S. WO/2006/091158 A1 (2006).

21. Nisman B, Allweis T, Kadouri L et al. Comparison of diagnostic and prognostic performance of two assays measuring thymidine kinase 1 activity in serum of breast cancer patients. Clin. Chem. Lab. Med. 51(2), 439-447 (2013).

22. Alegre MM, Weyant MJ, Bennett DT et al. Serum detection of thymidine kinase 1 as a means of early detection of lung cancer. Anticancer Res. 34, 2145-2152 (2014).

23. He Q, Wang N, Skog S, Ericsson S, Tribukait B. Characterization of a peptide antibody against a C-terminal part of human and mouse cytosolic thymidine kinase, which is a marker for cell proliferation. Eur. J. Cell Biol. 70(2), 117-124 (1996).

-. Basic article on the development of immunoassays directed against the 210 epitope.

24. He Q, Zou L, Zhang P, Liu J, Skog S, Fonander T. The clinical significance of thymidine kinase 1 measurement in serum of breast cancer patients using anti-TK1 antibody. Int. J. Biol. Markers 15, 139-146 (2000).

25. Kiran Kumar J, Aronsson A-C, Pilko G et al. A clinical evaluation of the TK 210 ELISA in sera from breast cancer patients demonstrates high sensitivity and specificity in all stages of disease. Tumour Biol. 37(9), 11937-11945. (2016).

26. He Q, Mao Y, Wu J et al. Cytosolic thymidine kinase is a specific histopathologic tumour marker for breast carcinomas. Int. J. Oncol. 25(4), 945-53 (2004).

- Comparison of Ki67 and TK1 immunostaining in malignant tissues.

27. Xu Y, Liu B, Shi Q-L et al. Thymidine kinase 1 is a better prognostic marker than Ki-67 for $\mathrm{pT} 1$ adenocarcinoma of the lung. Int. J. Clin. Exp. Med. 7(8), 2120-2128 (2014).

- Comparison of Ki67 and TK1 immunostaning in malignant tissues.

28. He Q, Mao Y, Wu J et al. Cytosolic thymidine kinase is a specific histopathologic tumour marker for breast carcinomas. Int. J. Oncol. 25(4), 945-53 (2004).

29. Chen Z, Zhou H, Li S et al. Serological thymidine kinase 1 (STK1) indicates an elevated risk for the development of malignant tumours. Anticancer Res. 28, 3897-3908 (2008)

-• An elevated serum TK1 concentration indicates an increased risk of a pathological condition, but not necessarily tumors.

30. Rehn S, Gronowitz JS, Källander C, Sundström C, Glimelius B. Deoxythymidine kinase in the tumour cells and serum of patients with non-Hodgkin lymphomas. British J. Cancer 71, 1099-1105 (1995).

31. Källander CFR, Simonsson B, Hagberg H, Gronowitz JP. Serum deoxythymidine kinase gives prognostic information in chronic lymphocytic leukemia. Cancer 54, 2450-2455 (1984).

•• Serum TK1 activity and disease progression in CLL.

32. Källander CFR, Simonsson B, Gronowitz JS, Nilsson K. Serum deoxythymidine kinase correlates with peripheral lymphocyte thymidine uptake in chronic lymphocytic leukemia. Eur. J. Haematol. 8(4), 331-337 (1987).

33. Procházka V, Faber E, Raida L, Langová K, Indrák K, Papajík T. High baseline serum thymidine kinase 1 level predicts unfavorable outcome in patients with follicular lymphoma. Leuk. Lymphoma 53(7), 1306-1310 (2012).

34. Hallek M, Langenmayer I, Nerl C et al. Elevated serum thymidine kinase levels identify a subgroup at high risk of disease progression in early, nonsmoldering chronic lymphocytic leukemia. Blood 93(5), 1732-1737 (1999).

35. Stelmach P, Błoński JZ, Wawrzyniak E et al. Prognostic value of thymidine kinase activity in patients with chronic lymphocytic leukemia. Postepy. Hig. Med. Dosw. 70, 1321-1330 (2016).

36. O'Neill KL, Zhang F, Li H, Fuja DG, Murray BK. Thymidine kinase 1 - a prognostic and diagnostic indicator in ALL and AML patients. Leukemia 21, 560-563. (2007).

-• Serum TK1 activity and disease progression in acute lymphoid leukemia and myelogenous leukemias.

37. Pan ZL, Ji XY, Shi YM, Zhou J, He E, Skog S. Serum thymidine kinase 1 concentration as a prognostic factor of chemotherapy-treated non-Hodgkin's lymphoma patients. TK1 levels before and 28 days after therapy are correlate with clinical response and 5-year survival. $J$. Cancer Res. Clin. Oncol. 136(8), 1193-1199 (2010).

38. Xu W, Cao X, Miao KR et al. Serum thymidine kinase 1 concentration in Chinese patients with chronic lymphocytic leukemia and its correlation with other prognostic factors. Int. J. Hematol. 90(2), 205-211. (2009). 
39. Unpublished Observations at AroCell.

40. Jagarlamudi KK, Kumer K, Fabjan T, Osredkar J, Eriksson S, Zupan M. Significance of TK 210 Protein (TK1) levels in lymphoma and leukemia patients. Poster at Immuno Oncology World Congress, Philadelphia, USA (2017).

41. Jagarlamudi KK, Eriksson S, Ulfstedt JM, Venge P, Molin D. The Thymidine kinase 1 (TK1) protein concentration in serum of Hodgkin Lymphoma patients; a marker of cell death and disruption of malignant cells? Poster Presented at the American Association for Cancer Research Annual Meeting 2018, IL, USA (2018).

42. Alegre MM, Robison RA, O’Neill KL. Thymidine kinase 1 upregulation is an early event in breast tumor formation. J. Oncol. 2012, 5 (2012).

- Increased TK1 expression may be an early event in the development of tumors.

43. Liu Y, Ling Y, Qi Q et al. Changes in serum thymidine kinase 1 levels during chemotherapy correlate with objective response in patients with advanced gastric cancer. Exper. Ther. Med. 2, 1177-1181, (2011).

44. Fujiwaki R, Hata K, Nakayama $\mathrm{K}$ et al. Thymidine kinase in epithelial ovarian cancer: relationship with the other pyrimidine pathway enzymes. Int. J. Cancer 99(3), 328-335. (2002).

45. Wang Y, Jiang X, Dong S, Shen J et al. Serum TK1 is a more reliable marker than CEA and AFP for cancer screening in a study of 56,286 people. Cancer Biomark. 16(4), 529-536 (2016).

46. Chen Y, Ying M, Chen Y et al. Serum thymidine kinase 1 correlates to clinical stages and clinical reactions and monitors the outcome of therapy of 1247 cancer patients in routine clinical settings. Int. J. Clin. Oncol. 15(4), 359-368. (2010).

47. Lou X, Zhou J, Ma Het al. The half-life of serum thymidine kinase 1 concentration is an important tool for monitoring surgical response in patients with lung cancer: a meta-analysis. Genet. Test Mol. Biomarkers 21(8), 471-478 (2017).

48. Jagarlamudi KK, Eriksson S, Zupan M et al. AroCell TK 210 ELISA May Complement Pro PSA and the Prostate Health Index in Differentiating Non-Cancerous from Cancerous Conditions in Prostate Disease. Poster presented at the UK National Cancer Research Institute Congress, Liverpool. BT Convention Center, Liverpool, UK, 5-8 November 2017.

49. He Q, Fornander $\mathrm{T}$, Johansson $\mathrm{H}$ et al. Thymidine kinase 1 in serum predicts increased risk of distant or loco-regional recurrence following surgery in patients with early breast cancer. Anticancer Res. 26, 4753-4760 (2006).

50. Jiang ZF, Wang M, Xu JL et al. Thymidine kinase 1 combined with CEA, CYFRA21-1 and NSE improved its diagnostic value for lung cancer. Life Sci. 194, 1-6 (2018).

-. Indicates the value of combining TK1 with other biomarkers.

51. Miran T, Vogg ATJ, El Moussaoui L et al. Dual addressing of thymidine synthesis pathways for effective targeting of proliferating melanoma. Cancer Med. 6(7), 1639-1651 (2017).

52. Barth R, Yang W, Wua G et al. Thymidine kinase 1 as a molecular target for boron neutron capture therapy of brain tumors. PNAS 105(45), 17493-17497 (2008).

-• Demonstrates the increased resistance on low TK1 bearing tissues to boron neutron therapy.

53. Weagel EG, Meng W, Townsend MH et al. Biomarker analysis and clinical relevance of TK1 on the cell membrane of Burkitt's lymphoma and acute lymphoblastic leukemia. Onco. Targets Therapy 10, 4355-4367. (2017).

54. Bading JR, Shields AF. Imaging of cell proliferation: status and prospects. J. Nucl. Med. 49, 64S-80S (2008).

-. Studies indicating how $\left[{ }^{18} \mathrm{~F}\right]$-FLT PET could potentially be used to study therapy response.

55. McKinley ET, Ayers GD, Smith RA et al. Limits of $\left[{ }^{18} \mathrm{~F}\right]-$ FLT PET as a biomarker of proliferation in oncology. PLoS ONE 8(3), e58938 (2013).

-. A review of the advantages and challenges of using $\left[{ }^{18}\right.$ F]-FLT PET.

56. Lee JH, Moon DH, Jung JH. Assessment of thymidine kinase 1 activity with 3 '-deoxy-3' - $\left.{ }^{18} \mathrm{~F}\right]$ fluorothymidine positron emission tomography in mice bearing human colon cancer xenografts after capecitabine treatment. J. Nucl. Med. 56 (Suppl. 3), 1182 (2015).

57. Schelhaas S, Heinzmann K, Bollineni VR et al. Preclinical applications of $3^{\prime}$-deoxy- $3^{\prime}-\left[{ }^{18} \mathrm{~F}\right]$ fluorothymidine in oncology - a systematic review. Theranostics 7(1), 40-50 (2017).

58. Rasey JS, Grierson JR, Wiens LW, Kolb PD, Schwartz JL. Validation of FLT uptake as a measure of thymidine kinase-1 activity in A549 carcinoma cells. J. Nuc. Med. 43(9), 1210-1217 (2002).

59. Hong IK, Kim SY, Chung JH et al. $3^{\prime}$-Deoxy $-3^{\prime}-\left[{ }^{18} \mathrm{~F}\right]$ fluorothymidine positron emission tomography imaging of thymidine kinase 1 activity after 5-fluorouracil treatment in a mouse tumor model. Anticancer Res. 34, 759-766 (2014).

60. Grem JL, Danenberg KD, Behan K et al. Thymidine kinase, thymidylate synthase, and dihydropyrimidine dehydrogenase profiles of cell lines of the National Cancer Institute's anticancer drug screen. Clin. Cancer Res. 7(4), 999-1009, (2001).

61. Kiran Kumar J, Wang L, Eriksson S. Doxorubicin effects on leukemia and breast cancer cells in culture on the TK1 protein levels using AroCell TK 210 ELISA: a tool for drug development. The 2017 Purine and Pyrimidine Society Meeting PP17 Gdańsk, Poland 20-24 September 2017.

•. The basic article on using TK1 immunoassy to study anticell proliferation effects in vivo. 
62. Bagegni N, Thomas $\mathrm{S}$, Liu $\mathrm{N}$ et al. Serum thymidine kinase 1 activity as a pharmacodynamic marker of cyclin-dependent kinase 4/6 inhibition in patients with early-stage breast cancer receiving neoadjuvant Palbociclib. Breast Cancer Res. 19(1), 123 (2017).

-. TK1 may be a particularly useful biomarker for studying the effects of this drug class.

63. Bonechi M, Galardi F, Biagioni C et al. Plasma thymidine kinase-1 activity predicts outcome in patients with hormone receptor-positive and HER2-negative metastatic breast cancer treated with endocrine therapy. Oncotarget 9(23), 16389-16399 (2018).

-• TK1 could be a particularly useful biomarker for for studying anti-hormonal therapy.

64. Gasparri F, Wang N, Skog S, Galvani A, Eriksson S. Thymidine kinase 1 expression defines an activate G1 state of the cell cycle as revealed with site-specific antibodies and ArrayScan assays. Eur. J. Cell. Biol. 88, 779-785 (2009).

65. Foekens JA, Romain S, Look MP, Martin P-M, Klijn JGM. Thymidine kinase and thymidylate synthase in advanced breast cancer: response to tamoxifen and chemotherapy. Cancer Res. 61, 1421-1425 (2001).

-. Tumor TK1 levels may provide information on therapy response and could aid in guiding therapy.

66. Tribukait B, Jagarlamudi K, Bergh J, Hatschek T. Quantitation of cell loss in breast cancer during neoadjuvant treatment (NACT) assessed by serum thymidine kinase protein concentration (sTK1). ESMO Asia Singapore 17-19 November 2018, Poster No 81P.

67. Li Z, Wang Y, Ma J et al. Transient increase in serum thymidine kinase 1 within one week after surgery of patients with carcinoma. Anticancer Res. 30(4), 1295-1299 (2010).

- Indicates that the transient increases seen in serum TK1 following therapy may not always be derived from the malignancy.

68. Zou L, Zhang PG, Zou S, Li Y, He Q. The half-life of thymidine kinase 1 in serum measured by ECL dot blot: a potential marker for monitoring the response to surgery of patients with gastric cancer. Int. J. Biol. Markers 17(2), 135-140 (2002).

- Information on the decline of serum TK1 immunoreactivity following therapy.

69. Nisman B, Appelbaum L, Yutkin V et al. Serum thymidine kinase 1 activity following nephrectomy for renal cell carcinoma and radiofrequency ablation of metastases to lung and liver. Anticancer Res. 36(4), 1791-1797 (2016).

- Information on the decline of serum TK1 enzyme activity following therapy.

70. Van Poznak C, Somerfield MR, Bast RC et al. Use of biomarkers to guide decisions on systemic therapy for women with metastatic breast cancer: American society of clinical oncology clinical practice guideline. J. Clin. Oncol. 33(24), 2695-2704 (2015).

71. Mottet N, Bastian PJ, Bellmunt J et al. Guidelines on prostate cancer. Eur. Assoc. Urol. 65(1), 124-137 (2014). 